\title{
Archaeal virus escapology
}

9

the electron

tomography

images reveal

a budding

mechanism

of egress by

an enveloped

archaeal virus

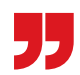

Archaea are infected by viruses that have remarkably diverse morphologies compared with eukaryotic viruses or phages (that is, bacterial viruses). Unlike the vast majority of phages, many archaeal viruses are enveloped by a lipid membrane. However, although many enveloped eukaryotic viruses - including HIV, influenza virus and Ebola virus - have been studied in detail, little is known about enveloped viruses that infect archaea. Using electron microscopy techniques, Quemin et al. were able to visualize the morphogenesis and egress of a prototypical enveloped archaeal virus, revealing some key similarities with eukaryotic viruses.

One of the most abundant and widespread family of archaeal viruses is the Fuselloviridae, of which Sulfolobus spindle-shaped virus 1 (SSV1) is the prototypical member. As suggested by its name, the SSV1 virion has a spindle-shaped morphology; the spindle is formed by five structural proteins, one of

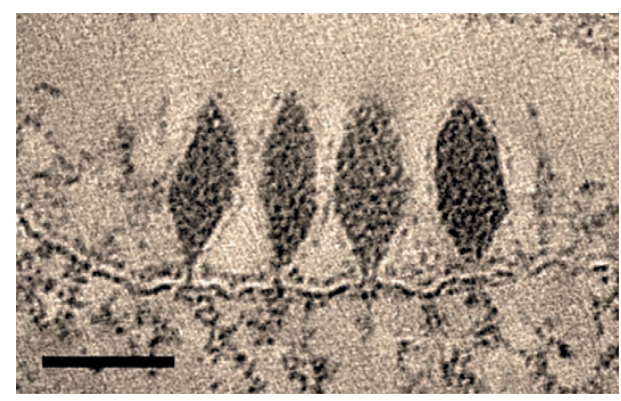

A slice through a tomogram that shows SSV1 virions attached to the cell surface (bar, $50 \mathrm{~nm}$ ). Image courtesy of P. Chlanda, US National Institutes of Health, Bethesda, Maryland, USA. which is host-encoded, and a circular double-stranded DNA genome. Biochemical analyses have previously demonstrated that the virus is enveloped by host-derived lipids.

To study the morphogenesis and egress mechanisms of SSV1, the authors used dual-axis electron tomography to image virions during infection of Sulfolobus shibatae cells. In common with many archaea, the $S$. shibatae cell surface has an S-layer, which is a cell-wall-like layer of glycoproteins that surrounds the membrane. The membrane itself is composed of a single monolayer of ether-based glycolipids. Thus, the physical barriers to egress are substantially different to those encountered by enveloped eukaryotic viruses. The tomography images suggested that virus assembly occurs at the host cell plasma membrane, which formed bulges around electron densities that were thought to correspond to virions. Interestingly, two morphologies were observed: the expected spindle shape, which is present in mature virions, and a novel rod-shaped intermediate. Maturation from rod to spindle was concomitant with constriction of the budding membrane, which occurred at the trailing end of the virion-containing bud.

How scission occured was unclear — it did not correlate with virus maturation, which suggests that host factors may instead be responsible. The authors note that viral egress by some enveloped eukaryotic viruses relies on endosomal sorting complex required for transport (ESCRT) proteins, which are also found in S. shibatae. Intriguingly, in a small number of samples, ring-like structures were observed that could be consistent with constriction at the neck of the bud by a helical ESCRTlike polymer. As egress involves the unusual step of scission of a single monolayer of ether-based lipids, the future elucidation of this mechanism will be of interest. Following scission, virions remained trapped on the surface; this suggests that membrane scission is insufficient for virus egress, probably owing to the small size of the pores in the S-layer, which are narrower than the virion. A change in S-layer organization was observed and, following local host membrane ruptures, virions were eventually released. Thus, SSV1 seems to locally disrupt the S-layer to complete egress.

Together, the electron tomography images reveal a budding mechanism of egress by an enveloped archaeal virus that is reminiscent of egress by enveloped eukaryotic viruses. Whether the similarity extends to a common molecular mechanism, or is instead superficial (and based on a novel molecular mechanism), will await future studies.

Naomi Attar

ORIGINAL ARTICLE Quemin, E. R. et al. Eukaryotic-like virus budding in archaea. $m$ Bio 7 , e01439-16 (2016) 